\title{
A Chronicle of Analyzing Stochasticity in Multi Period Transportation Problems for Uncertainty
}

\author{
Sajal Chakroborty \\ Department of Electronics and Communications \\ Engineering \\ East West University \\ Dhaka, Bangladesh
}

\author{
M. Babul Hasan \\ Department of Mathematics \\ University of Dhaka Bangladesh
}

\begin{abstract}
Objective of this paper is to develop a new technique for solving Transportation Problems (TP) by considering uncertainty in deterministic case for multi periods. There are many obtainable techniques for solving TP for deterministic cases. But these techniques are not applicable to solve multi period TP when uncertainty arises. In this paper, a technique has proposed to handle this uncertainty for different scenarios. To develop the technique, idea of decomposition based pricing (DBP) method has used. A real life oriented problem has developed by collecting data from a business organization of Bangladesh and has analyzed by the proposed technique.
\end{abstract}

\section{Keywords}

SP, TP, AMPL, DBP.

\section{INTRODUCTION}

Uncertainty is a common matter in real life. It can occur anywhere in anytime. Stochastic programming (SP) is a special type of mathematical programming that deals with such uncertainty [1-2]. The beginning of SP dates back to the 50 's and 60's of the last century. G. B. Dantzig first formulated the general problem of LP with uncertain data and he is considered as a pioneer to establish SP as a branch of mathematics. The main advantage of SP is that it can estimate the probability of occurring different scenarios for respectable uncertainty.

Transportation problem (TP) deals with the situation in which commodities are shifted from sources to destinations in such a way that the demand of customers and supplying capabilities of sources are satisfied within minimum cost. It can be considered as a relation between sources and destinations. It was first formulated by French mathematician Grapard Monge in 1781. But major advances in this field were made during World War II by the Russian mathematician Leonid Kantorvich. Idea of TP can be easily extended to apply in the area of inventory control, employment scheduling, personnel assignment, cash flow statements and many others.

Normally TP is a deterministic case. By the developed technique it can be solved when uncertainty arises.

The paper has been outlined like: in Section 2, some basic ideas and preliminaries about stochastic programming (SP) and transportation problems (TP) relevant to the work has been discussed. In Section 3, some existing techniques for solving SP and TP problems have been presented. In Section 4 , a stochastic multi period TP model has been developed and in Section 5, its details formulation has been presented. In Section 6, an algorithm has developed for solving multi period TP problems and in Section 7, computer code has been developed. In Section 8, details optimal solution with discussion ha presented and then in Section 9, a graphical comparison between profits has presented. Finally in Section 10 , a conclusion has drawn about the work.

\section{PRELIMINARIES}

In the current section, some basic definitions and relevant techniques for solving TP and SP problems have been discussed.

\subsection{Stochastic Programming}

Consider the following linear programming (LP) problem [3].

Maximize (or Minimize)

$$
z=\sum_{i=1}^{n} c_{i} x_{i}
$$

subject to

$$
\begin{aligned}
& \sum_{i=1}^{n} a_{i j} x_{i}\{\leq,=, \geq\} b_{j}, j=1, \ldots, m \\
& x_{i} \geq 0, i=1, \ldots, n
\end{aligned}
$$

Where one and only one of the signs $\leq,=, \geq$ will hold in equation (2) and the sign may vary from one constraint to another. Here $c_{i}(i=1 . . n)$ are called profit or cost coefficients, $b_{j}(j=1, \ldots, m)$ are called right hand side constraint and $x_{i}(i=1 . . n)$ are called the decision variables. The set of feasible solutions to LP is written as $\mathrm{S}=$ $\left\{\left(x_{1}, x_{2}, \ldots, x_{n}\right)^{T}:\left(x_{1}, x_{2}, \ldots, x_{n}\right)^{T} \in R^{n}\right\}$. When all or some of the parameters of $\mathrm{c}_{\mathrm{i}}, b_{j}, a_{i j}$ contain uncertainty then it is called stochastic programming (SP) problem. It can be written mathematically as follows [4].

Maximize (or Minimize)

$$
z=\sum_{i=1}^{n} P_{\omega} c_{\omega i} x_{\omega i}
$$

subject to

$$
\begin{gathered}
\sum_{i=1}^{n} a_{i j} x_{\omega i}\{\leq,=, \geq\} b_{j}, j=1, \ldots, m \\
x_{\omega i} \geq 0, i=1, \ldots, n
\end{gathered}
$$

In this definition, $c_{i}$ has considered as an uncertain parameter and scenarios $\omega$ are inserted in it. $x_{\omega i}$, where $i=1, \ldots, n$ are the stochastic decision variables and $P_{\omega}$ is the probability for scenarios $\omega$. 


\subsection{Scenarios}

A scenario for a stochastic model is a collection of outcomes for all the stochastic events taking place in the model, along with the associated probability of the scenario to occur. Khan and Weiner (1967), probably first originated scenario analysis [5], defined a scenario as a hypothetical sequence of events constructed to focus on casual processes and decision points. Scenarios can be of several types, the most common being "favorability to sponsor," e.g. optimistic or pessimistic and "Probability of occurrence," e.g. most likely or least likely, is also very popular although very subjective. Sources of data for scenarios are historical data, expert opinions, simulation based on a mathematical or statistical model.

\subsection{Transportation Problem}

It is a special type of linear programming problem (LP). It can be formulated as an LP problem as follows [6].

$$
\begin{gathered}
\text { Minimize } z=\sum_{i=1}^{m} \sum_{j=1}^{n} c_{i j} x_{i j} \\
\text { subject to } \\
\sum_{j=1}^{n} x_{i j} \leq a_{i}(i \text { for } i=1,2, \ldots . m) \\
\sum_{i=1}^{m} x_{i j} \geq b_{j}(j \text { for } j=1,2, \ldots . \mathrm{n}) \\
\left.x_{i j} \geq 0 \text { (Nonnegative restrictions for all pairs }(i, j)\right)
\end{gathered}
$$

Here $x_{i j}$ is representing the commodity shipped from source $i$ to destination $j$ and $a_{i}$ is the amount of commodity shipped from source $i, b_{j}$ is the amount of commodity demanded at destination $j$. When total amount of commodity shipped and total of that is demanded become same, the problem is called balanced TP and when are not same it is called unbalanced TP.

\subsection{Decomposition Based Pricing}

This technique was developed by Mamer and McBride [7]. In this section, we have discussed decomposition based pricing algorithm briefly in the following steps.

Step 1: Relax complicating constraints by subtracting from objective function of the original problem. Decompose the whole problem into sub-problems and a master problem. Solve sub-problems and generate muster problem by deleting those variables which do not provide non negative values from the original problem.

Step 2: Stop when sub problem value and master problem value become equal. Otherwise repeat the previous steps.

\section{EXISTING TECHNIQUES}

In this section, some existing techniques have been discussed briefly for solving SP and TP problems.

\subsection{L-Shaped Method}

This method was developed by Slyke and Wets [8]. It was also developed by using a decomposition technique named Bender's decomposition. It contains the following steps.

Step 1: It decomposes the whole problem into two stages first. The first stage problem leads to a master problem and the second stage problem leads to a sub-problem.
Step 2: The second stage objective function is approximated by using a piecewise linear convex function.

Step 3: Then approximation is developed iteratively, and convex function is typically represented in a master program using a cutting plane approximation.

One of the drawbacks of this method is that, when the number of scenarios is much too high it's quite difficult to solve the problem by using this method.

\subsection{Nested Decomposition Technique}

It is a solution procedure of multi-stage SP problems. It just likes a recursive version of L-shaped method [9]. In this procedure, a problem mainly solved sequentially and it can be represented by a scenario tree representation with the change of associated probability with time. It has been discussed briefly in the following steps [10].

Step 1: Parent nodes send proposals for solutions to their children nodes.

Step 2: Child nodes send cuts to their parent nodes.

Step 3: There are different "sequence procedures" that tell in which order the problems corresponding to different nodes in the scenario tree are solved.

\subsection{North West Corner Rule}

Step 1: Select the upper left (North West) cell of the transportation table.

Step 2: If allocation made is equal to the supply available at the first source (in the first row) then move vertically down to the next cell.

If allocation made is equal to the demand of the first destination (in the first column) then move horizontally to the next cell.

Step 3: Continue this process until an allocation is made in the south east corner cell of the transportation table.

The basic solutions obtained by this method may be far from optimal since the transportation costs are completely ignored [11].

\subsection{Vogel's Approximation Method}

Step 1: Calculate penalty for each row and column by taking the difference between the two smallest unit costs. This penalty or extra cost has to be paid if one fails to allocate the minimum unit cost.

Step 2: Select the row or column with the highest penalty or select the minimum unit cost of that row or column. Then allocate the minimum of supply or demand values of that cell. If there is a tie then select the cell where maximum allocation could be made.

Step 3: Adjust the supply or demand and eliminate the satisfied row and column. If a row and column are satisfied simultaneously, one of them is eliminated and the other one is assigned to zero value. Any row or column having zero supply or demand cannot be used in calculating future penalties.

Step 4: Repeat this process until all the supply sources and demand destinations are satisfied.

This method yields a better initial solution which sometimes may be the optimal solution. 
In the next section, a stochastic multi period TP model has presented. This model has developed by collecting data from a business organization of Bangladesh.

\section{A STOCHASTIC MULTI PERIOD TP MODEL}

"Ahmed Textile Ltd" is one of the renowned garment industries in Bangladesh. They have four garment factories and they supply their products in different markets all over the country. They want to maximize their profit by minimizing their transportation cost. But their profit can change due to some uncertainties such as political conditions, lack of raw materials, demand of customers etc.

In this model, eight different markets in Dhaka city have considered where products of the company are supplied and have considered three different scenarios such as good, normal, and bad political conditions to analyze the profit. Here bad political condition means occurrences of hartals, strike of opposition parties, normal political condition means abating situations of such obstacles and good political condition means no such obstacles. Data for year 2014 have collected and the whole year has been divided into three different periods. Each period consists of four months such as from January to April as Period 1, May to August as Period 2, and September to December as Period 3. Due to volume whole data are not presented in this paper. If readers are interested then contact with the authors.

\section{FORMULATION OF THE MODEL}

In this section, the stochastic model presented in Section 4 has been formulated mathematically. Before formulation, basic notations and parameters have been discussed. Uncertainty has inserted into the cost parameter for transportation. Supply and demand remains fixed i.e. these are considered as deterministic parameters. Here profit has been calculated by subtracting minimum transportation cost from selling price.

Indices

- $\quad m$, number of sources.

- $\quad n$, number of destinations.

- $\omega$, number of scenarios.

- $t$, number of time periods.

Stochastic Parameters

- $\boldsymbol{c}_{\boldsymbol{\omega} i \boldsymbol{j} \boldsymbol{t}}$, transportation costs for different scenarios $\omega$, sources $i=1, \ldots, m$ and destinations $j=1, \ldots, n$.

- $\boldsymbol{P}_{\boldsymbol{\omega}}$, probability for different scenarios $\omega$.

Stochastic Decision Variables

- $\boldsymbol{x}_{\boldsymbol{\omega} i \mathrm{it}}$, products transported for different scenarios $\omega, \quad$ sources $i=1, \ldots, m \quad$ and destinations $j=1, \ldots, n$.

\section{Deterministic Parameters}

- $\boldsymbol{a}_{i t}$, amount of products supplied from different sources $i=1, \ldots, m$ in different periods $t$.

- $b_{j t}$, amount of products demanded in different destinations $j=1, \ldots, n$ in different periods $t$.

Here total amount of supplies of different products are same to total demands.

\section{Formulation}

$$
\text { Minimize } z=\sum_{i=1}^{m} \sum_{j=1}^{n} \sum_{t=1}^{T} P_{\omega} c_{\omega i j t} x_{\omega i j t}
$$

subject to

$$
\begin{gathered}
\sum_{i=1}^{m} \sum_{j=1}^{n} \sum_{t=1}^{T} x_{\omega i j t} \leq \sum_{i=1}^{m} \sum_{t=1}^{T} a_{i t} \\
\sum_{i=1}^{m} \sum_{j=1}^{n} \sum_{t=1}^{T} x_{\omega i j t} \geq \sum_{j=1}^{n} \sum_{t=1}^{T} b_{j t} \\
x_{\omega i j t} \geq 0
\end{gathered}
$$

\section{PROPOSED ALGORITHM}

In the current section, the proposed algorithm has been presented. Idea of DBP algorithm has used to develop this technique. It consists of the following steps.

Step 1: $k \leftarrow$ set number of iterations. Initially set $k=1$.

Step 2: Pick an initial set of prices $\lambda^{k}$ and this can be chosen by starting with $\lambda^{1}=0$. Or, start with $\lambda^{1}>0$ as the dual prices from the relaxed constraints of the LP relaxation.

Step 3: Solve the sub-problems,

$S\left(x, \lambda^{k}\right): \quad$ Minimize

$$
\sum_{j=1}^{n} \sum_{t=1}^{T}\left(\sum_{i=1}^{m} P_{\omega} c_{\omega i j t}-\lambda^{k}\right) x_{\omega i j t}+\lambda^{k} \sum_{j=1}^{n} \sum_{t=1}^{T} a_{j t}
$$

subject to

$$
\sum_{i=1}^{m} \sum_{t=1}^{T} x_{\omega i j t} \geq \sum_{t=1}^{T} b_{j t}
$$

$$
x_{\omega i j t} \geq 0, \text { where } t=1, \ldots, T, j=1, \ldots, n
$$

Here sub-problem has generated by considering the supply constrains as complicating constraint. It is also possible to generate a sub-problem by subtracting some or all demand constraints from the objective function.

Step 4: Master problem will be generated from original problem by deleting those variables which do not have non negative values. Solve the following restricted master problem.

$M\left(x, \lambda^{k}\right):$ Minimize,

$$
Z=\sum_{i=1}^{m} \sum_{j=1}^{n} \sum_{t=1}^{T} P_{\omega} c_{\omega i j t} x_{\omega i j t}
$$

subject to

$$
\begin{gathered}
\sum_{j=1}^{n} \sum_{t=1}^{T} x_{\omega i j t} \leq \sum_{i=1}^{m} \sum_{t=1}^{T} a_{i t} \\
\sum_{i=1}^{m} \sum_{t=1}^{T} x_{\omega i j t} \geq \sum_{j=1}^{n} \sum_{t=1}^{T} b_{j t} \\
x_{\omega i j t} \in I
\end{gathered}
$$

Here $I$ is a non empty set of all variables which give non negative values in sub problems. 
Step 5: For stopping criterion, two alternations are used.

(i) Stop when the objective value of the sub-problem and the restricted master problem are equal i.e. $v\left(S^{k}\right)=v\left(M^{k+1}\right)$. Else set $\mathrm{k}=\mathrm{k}+1$, go to step- 1 . Here $v\left(S^{k}\right)$ and $v\left(M^{k+1}\right)$ are representing sub problem and master problem values respectively. Or,

(ii) Stop when no new variables come into the restricted master. Else go to step-2.

\section{COMPUTER CODE}

It is noted that, the problem presented in Section 5 is a four dimensional vector and it is very difficult to solve manually. That's why in this section, a computer code has been developed by using a mathematical programming language (AMPL) according to the proposed algorithm. The code consists of three different parts. These are AMPL model file, data file and run file. Due to volume it has not presented in this paper. If readers are interested then they can contact with authors.

In the next section, the optimal solution of the stochastic model has presented.

\section{OPTIMAL SOLUTION AND PROFIT CALCULATION}

In the current section, profits have been calculated and optimal solutions are presented for different periods and scenarios. Profits have been calculated by subtracting minimum transportation costs obtained by our code from selling prices survived by the company. Selling prices are considered to be remaining fixed for different periods but it varies for different scenarios. The result has been presented in the following tables.

Table 1. Profit for Period 1

\begin{tabular}{|c|c|c|c|}
\hline & $\begin{array}{c}\text { Selling } \\
\text { Prices } \\
\text { (TK) }\end{array}$ & $\begin{array}{c}\text { Minimum } \\
\text { transportation } \\
\text { costs (Tk) }\end{array}$ & Profit (Tk) \\
\hline Scenario 1 & 20000 & 7135 & 12865 \\
\hline Scenario 2 & 25000 & 2738 & 22262 \\
\hline Scenario 3 & 30000 & 2364 & 27636 \\
\hline
\end{tabular}

From the above table, it can be observed that maximum profit is TK.27, 636 has been obtained for good political condition. Maximum selling price has been obtained at that scenario compared to other scenarios. It can also be observed that transportation cost is minimum when political condition was good. In the next table, profits obtained for Period 2 has ben presented.

Table 2. Profit for Period 2

\begin{tabular}{|c|c|c|c|}
\hline & $\begin{array}{c}\text { Selling } \\
\text { Prices } \\
\text { (TK) }\end{array}$ & $\begin{array}{c}\text { Minimum } \\
\text { transportation } \\
\text { costs (Tk) }\end{array}$ & $\begin{array}{c}\text { Profit } \\
\text { (Tk) }\end{array}$ \\
\hline Scenario 1 & 20000 & 7915 & 12085 \\
\hline Scenario 2 & 25000 & 2840 & 22160 \\
\hline Scenario 3 & 30000 & 2208 & 27792 \\
\hline
\end{tabular}

From Table 2, it can be observed that minimum profit has been obtained due to the first scenario when transportation cost is greater than other scenarios and maximum profit has been obtained for good political condition and it is TK. 27792. In the next table, obtained profit with minimum transportation cost and selling price has been presented.

Table 3. Profit for Period 3

\begin{tabular}{|c|c|c|c|}
\hline & $\begin{array}{l}\text { Selling } \\
\text { Prices } \\
(\mathrm{TK})\end{array}$ & $\begin{array}{l}\text { Minimum } \\
\text { transportation } \\
\text { costs (Tk) }\end{array}$ & $\begin{array}{l}\text { Profit } \\
(\mathrm{Tk})\end{array}$ \\
\hline Scenario 1 & 20000 & 7485 & 12515 \\
\hline Scenario 2 & 25000 & 2368 & 22632 \\
\hline Scenario 3 & 30000 & 2271 & 27723 \\
\hline
\end{tabular}

From Table 3, it can be observed that the similar case like other different periods. Here maximum profit has also obtained for good political condition.

\section{PROFIT COMPARISIONS}

In this section, a comparison has been presented between profits for different scenarios and for different periods. This comparison has been presented graphically as follows.

\section{Profit Comparisons}

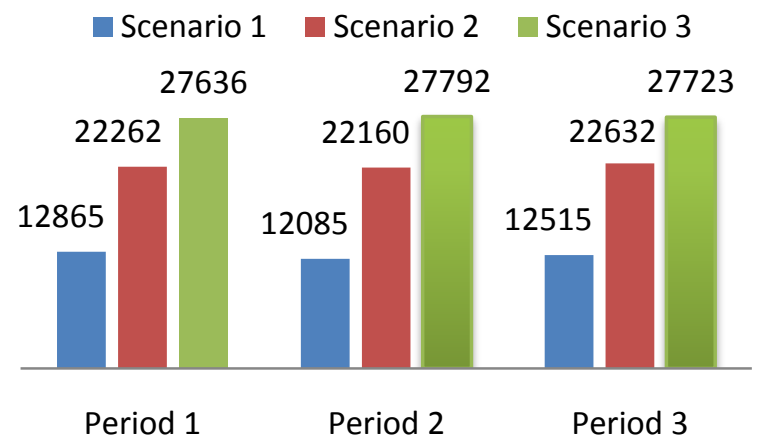

Fig.1: Profit comparisons

In Fig.1, blue charts are representing profits for bad political condition, red charts are representing for normal political condition, and green charts are representing profits for good political condition. From the above figure, it can be observed that highest profit is TK. 27,792 has made for good political condition at Period 2 and minimum profit is TK.12,085 has been obtained for bad political condition at same period comparing to others. From these result, we can make a forecast that for normal and good political condition the company need to be worried about the risk to make loss in business. But they have to make some plans when political condition is bad to abate the rate of loss. They have to wait and prepare so that at the due time of removing bad conditions they can make quick supplies and fulfill their market demands so that the situations arisen at the uncertain moment can be overcome. For this they must have a plan for making inventories and a preparation for own transportation system. 


\section{CONCLUSION}

In this paper, a new algorithm with computer code had presented for analyzing stochastic effect on multi period TP problems for uncertainty. A multi period TP model had developed by collecting sample data from a business organization. It was solved by using our developed AMPL code. An analysis about the fluctuation of profits due to different scenarios in different periods had shown. It is wished that this study can help an organization to make a future plan about their business and it will help them to make forecasts about future.

\section{ACKNOWLEDGEMENT}

Our thanks to the experts who have contributed towards the development of the work.

\section{REFERENCES}

[1] Domenica, N., G. Mitra and Valente, P., 2007. Stochastic Programming and Scenario Generation within a Simulation Framework: An Information Systems Perspective, Decision support system, vol.42 (4), pp.2197-2218.

[2] Mayer, J., and Kall, P., 1997.Stochastic Linear Programming, Springer.

[3] Taha, H. A., Operations research: An introduction, $8^{\text {th }}$ Ed. Pearson Princeton hall.

[4] Brige, J. R. and Louveaux, F., 1997. Introduction to Stochastic Programming, Springer-Verlag, New York .
[5] Weiner A., and Khan, H., "The Year 2000: A Framework for Speculation on the next Thirty Three years, Macmillan, New York (1967).

[6] Gupta, P.K., D.S. Hira, 2005.Problems in Operations Research Principles and Solution, S.Chand \& Company LTD., New Delhi-110055, 406-484.

[7] Mamer, J. W. \& R. D. McBride, 2000.A Decompositionbased Pricing Procedure for Large- Scale Linear Programs: An application to the linear multi-commodity Flow Problem, vol.46 (5), pp.693-709.

[8] Slyke, R. V., and Wets, R. G. B., 1969. L-Shaped Programs with Applications to Control and Stochastic Programming, SIAM, J. on Applied Mathematics, vol.17(4), pp.638-663.

[9] Linderoth, J. and Wright, S., 2003.Decomposition Algorithm on a computational grid, Comput. Optim. Appl., vol.24, pp.207-250.

[10] Higel, J.L., and Sen, S., 1999.Statistical Approximations for Stochastic Linear Programs, Ann. Oper. Res., vol.85(1). pp.173-192.

[11] Winston, W.L., 1994. Linear Programming: Applications and Algorithm, Dunbury Press, Bellmont, California, U.S.A. 